\title{
The Dynamics of Very High Alfvén Mach Number Shocks in Space Plasmas
}

\author{
Torbjörn Sundberg ${ }^{1}$, David Burgess ${ }^{1}$, Manfred Scholer $^{2}$, Adam Masters ${ }^{3}$, and Ali H. Sulaiman ${ }^{4}$ \\ ${ }^{1}$ School of Physics and Astronomy, Queen Mary University of London, London, E1 4NS, UK; torbjorn.sundberg@gmail.com \\ ${ }^{2}$ Max-Planck-Institut für extraterrestrische Physik, Garching, Germany \\ ${ }^{3}$ The Blackett Laboratory, Imperial College London, Prince Consort Road, London, SW7 2AZ, UK \\ ${ }^{4}$ Department of Physics and Astronomy, University of Iowa, Iowa City, Iowa 52242, USA \\ Received 2016 October 26; revised 2016 December 22; accepted 2016 December 23; published 2017 February 6
}

\begin{abstract}
Astrophysical shocks, such as planetary bow shocks or supernova remnant shocks, are often in the high or veryhigh Mach number regime, and the structure of such shocks is crucial for understanding particle acceleration and plasma heating, as well inherently interesting. Recent magnetic field observations at Saturn's bow shock, for Alfvén Mach numbers greater than about 25, have provided evidence for periodic non-stationarity, although the details of the ion- and electron-scale processes remain unclear due to limited plasma data. High-resolution, multispacecraft data are available for the terrestrial bow shock, but here the very high Mach number regime is only attained on extremely rare occasions. Here we present magnetic field and particle data from three such quasiperpendicular shock crossings observed by the four-spacecraft Cluster mission. Although both ion reflection and the shock profile are modulated at the upstream ion gyroperiod timescale, the dominant wave growth in the foot takes place at sub-proton length scales and is consistent with being driven by the ion Weibel instability. The observed large-scale behavior depends strongly on cross-scale coupling between ion and electron processes, with ion reflection never fully suppressed, and this suggests a model of the shock dynamics that is in conflict with previous models of non-stationarity. Thus, the observations offer insight into the conditions prevalent in many inaccessible astrophysical environments, and provide important constraints for acceleration processes at such shocks.
\end{abstract}

Key words: acceleration of particles

\section{Introduction}

Collisionless shocks are important in a wide range of astrophysical environments: at planetary magnetospheres in the solar system (e.g., Russell 1985), at the outer boundary of the heliosphere (e.g., Jokipii 2013), and in a variety of astrophysical contexts such as around supernova remnants (e.g., Helder et al. 2012; Bell 2013). In situ observations have long helped to shape our understanding of the physics acting in these shocks. These investigations have typically been focused on moderate to high Alfvén Mach numbers $\left(M_{\mathrm{A}} \sim 3-15\right.$, as found at the terrestrial bow shock), whereas our knowledge and understanding of the very high Mach number shock regime (e.g., $M_{\mathrm{A}}>25$ ) has mostly been restricted to numerical simulations, as these shocks are very rare in our near-space environment.

Collisionless shocks are characterized by a series of parameters, such as the Mach number (whether Alfvénic, fast or sonic), which is the ratio between the shock velocity in the upstream medium and the relevant wave velocity; the plasma $\beta$, which is the ratio between thermal and magnetic pressures; and the magnetic geometry of the shock, given as the angle between the upstream magnetic field and the shock normal, $\theta_{\mathrm{Bn}}$. In this context, quasi-perpendicular refers to a shock geometry with $\theta_{\mathrm{Bn}} \geqslant 45^{\circ}$, whereas lower $\theta_{\mathrm{Bn}}$ geometries are referred to as quasi-parallel shocks. Due to a fundamental difference in the trajectories of ions and electrons at the shock transition, these two shock configurations display very different shock dynamics, driven by ion- and electron-scale processes at the shock interface (see for example Burgess \& Scholer 2015 for details).

At quasi-perpendicular shocks, the average shock structure is dominated by a foot of reflected ions, which is upstream of the shock ramp where the major thermalization and deceleration occurs. Non-stationarity in the form of rippling of the surface or steepened whistler waves (Moullard et al. 2006; Lobzin et al. 2007) is an intrinsic feature of the shock, but this is generally manifest as minor perturbations on top of an otherwise stationary shock ramp. Simulations have predicted that if the fraction of ions reflected by the shock front becomes sufficiently high, the quasi-perpendicular shock can become periodically reforming on timescales of the ion gyroperiod. Various theories have been suggested for such non-stationarity, including self-reformation where a new shock ramp grows at the edge of the foot (Biskamp \& Welter 1972a; Lembège \& Dawson 1987), whistler-induced reformation (Biskamp \& Welter 1972b; Scholer \& Burgess 2007), kinetic instabilities such as the Buneman and modified two-stream instability (e.g., Cargill \& Papadopoulos 1988; Matsukiyo \& Scholer 2003, 2006b; Scholer et al. 2003; Scholer \& Burgess 2007; Matsumoto et al. 2013), and gradient catastrophe of nonlinear whistler waves due to steepening (Krasnoselskikh et al. 2002). However, it has not been until recently that such nonstationarity has been confirmed with in situ spacecraft observations. In a survey of Cassini shock crossings at Saturn, Sulaiman et al. (2015) found evidence of a periodically reforming shock, pulsating at a period near 0.3 of the ion gyroperiod in the unperturbed upstream medium. This period agrees with the time taken for a specularly reflected proton to gyrate across the foot and return to the main shock ramp. Sulaiman et al. (2015) also report that these periodic nonstationary shocks are primarily found in the very high Mach number regime, which gives evidence for a relation between Mach number and reformation. The main processes behind the non-stationary behavior of these very high Mach number 
Table 1

Shock Parameters of the Studied Events ${ }^{\mathrm{a}}$

\begin{tabular}{lllcccrr}
\hline \hline Date & $V_{\mathrm{sw}}$ & $B_{\mathrm{sw}}$ & $n_{\mathrm{sw}}$ & $\beta$ & $M_{\mathrm{A}}$ & $M_{\mathrm{S}}$ & $\theta_{\mathrm{Bn}}$ \\
\hline 2006 Mar 25 & $360 \mathrm{~km} \mathrm{~s}^{-1}$ & $2.5 \mathrm{nT}$ & $14.8 \mathrm{~cm}^{-3}$ & 11.9 & 24 & 8.3 & $45^{\circ}$ \\
2009 Feb 19 & $310 \mathrm{~km} \mathrm{~s}^{-1}$ & $1 \mathrm{nT}$ & $8.6 \mathrm{~cm}^{-3}$ & 33.7 & 39 & 7.9 & $85^{\circ}$ \\
2013 Mar 24 & $430 \mathrm{~km} \mathrm{~s}^{-1}$ & $1.4 \mathrm{nT}$ & $10.4 \mathrm{~cm}^{-3}$ & 22.5 & 41 & 11.1 & $45^{\circ}$ \\
\hline
\end{tabular}

Note.

${ }^{\mathrm{a}}$ For each event, the date, solar wind velocity, magnetic field, density, plasma $\beta$, and Alfvénic and sonic Mach numbers are shown, along with the angle between the shock normal and the upstream magnetic field $\left(\theta_{\mathrm{Bn}}\right)$ and upstream velocity $\left(\theta_{\mathrm{Vn}}\right)$.

C1
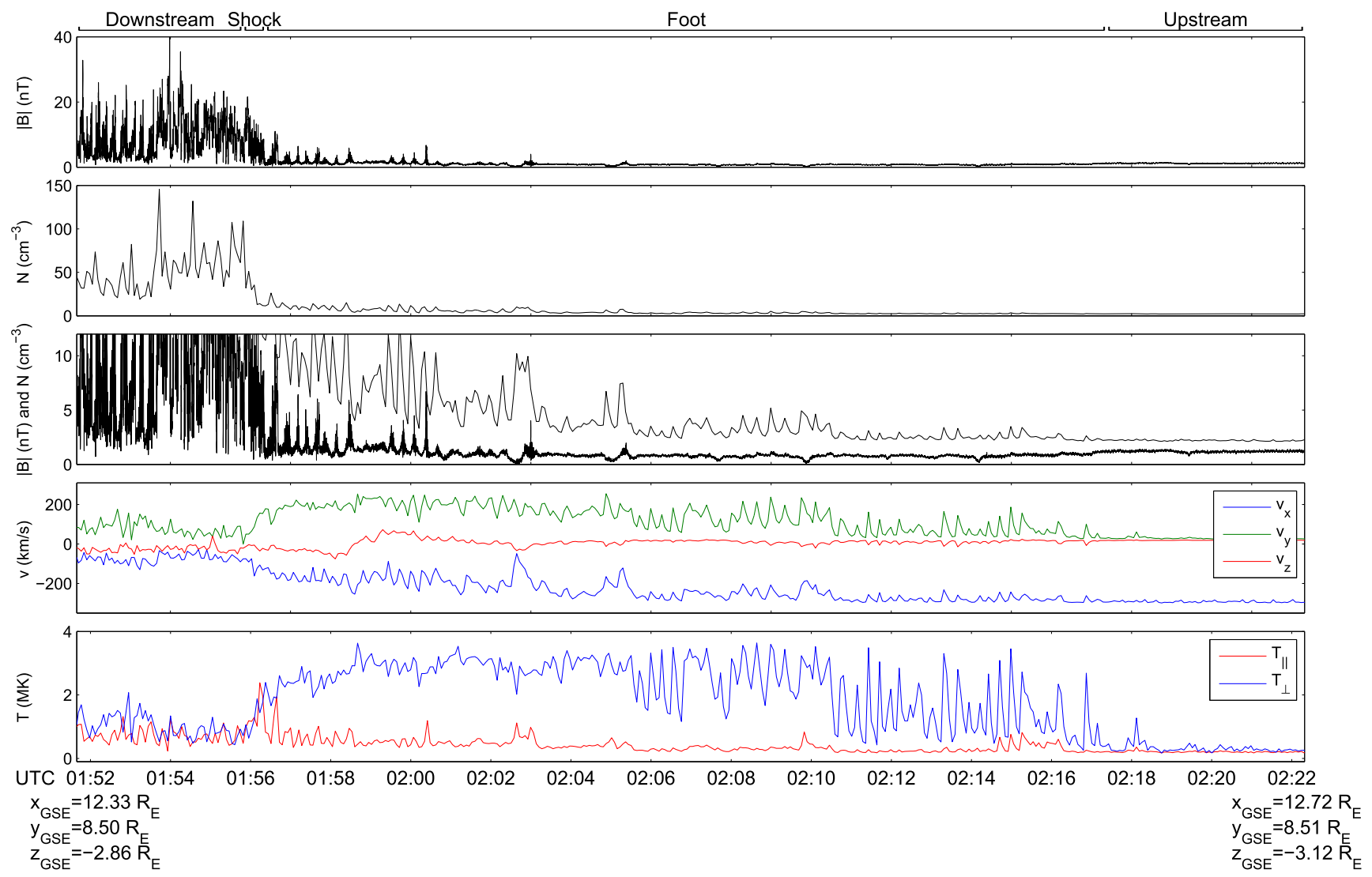

Figure 1. Overview of the foot of a high Mach number shock crossing, observed on 2009 February 19. The first three panels show the magnetic field magnitude, the number density, and the two quantities overlaid on a reduced scale to highlight low-level variations. The fourth panel shows the $x$ (blue), $y$ (green), and $z$ (red) components of the velocity, and the fifth panel shows the parallel (red) and perpendicular (blue) ion temperatures.

shocks, such as the details of the ion- and electron-scale processes acting within the shock transition, remain elusive.

\section{Observations}

Typically, Earth's bow shock has a shock strength in terms of Alfvén Mach number $M_{\mathrm{A}} \sim 5-10$; however, in very rare cases, it can attain a very high Mach number regime with $M_{\mathrm{A}} \geqslant 25$, in the range more often experienced at the outer planets, and approaching the range important for supernova remnant shocks. For these periods we can take advantage of the high-quality multi-spacecraft measurements available at Earth to investigate the detailed microphysics of the shock. The events in this study were selected from a data set of very high Alfvén Mach number intervals based on the five-minute resolution OMNI solar wind data set in combination with a survey of shock crossings and shock dynamics using data from the four-spacecraft Cluster mission. At Earth, conditions with
Mach numbers $M_{\mathrm{A}}>25$ are observed approximately $1 \%$ of the time, although most such intervals are short-lived and do not provide the stable conditions required to observe the ion-scale processes associated with the shock. In over 15 years of Cluster operations, we have found three events in the very high Mach number regime that show clear periodic non-stationary behavior. All three events are associated with both unusually high solar wind density and unusually weak magnetic field, and they all remain at a high Mach number level over several hours. The main parameters for these shocks are given in Table 1 .

Figure 1 gives an overview of the magnetic field $(B)$, velocity $(v)$, density $(N)$, and temperature $(T)$ of a very high Mach number shock observed by Cluster 1 on 2009 February 19. The magnetic field data in this study are provided by the Cluster Fluxgate Magnetometer (FGM; Balogh et al. 2001), the electron spectra from the Plasma Electron And Current Experiment (PEACE; Johnstone et al. 1997), and the ion 
velocity space distributions and moments from the Cluster Hot Ion Analyser of the Cluster Ion Spectrometry (CIS) experiment (Reme et al. 1997). The ion moments (density, temperature, and bulk velocity) are calculated on board the spacecraft over the $4 \mathrm{~s}$ spacecraft spin period, and 3D distributions are resolved in the $5 \mathrm{eV}-32 \mathrm{keV}$ range with $22^{\circ} .5$ resolution in both azimuth and elevation. The $x y z$ components are given in a geocentric solar ecliptic (GSE) coordinate system, with $x$ directed toward the Sun, $y$ toward dusk, and $z$ toward the ecliptic north pole. Parallel and perpendicular quantities relate to the background magnetic field. Due to the relative motion of the spacecraft and shock, the transition was observed from downstream to upstream, as marked. The foot of reflected ions is seen for more than 20 minutes following the shock crossing at 01:56 UT, and the ion properties change gradually as the spacecraft passes through the foot and into the pristine solar wind. During this period, the spacecraft moved outward at a velocity of $1.7 \mathrm{~km} \mathrm{~s}^{-1}$, from $x=12.33 R_{\mathrm{E}}$ and $z=-2.86 R_{\mathrm{E}}$ at $01: 52 \mathrm{UT}$ to $x=12.72 R_{\mathrm{E}}$ and $z=-3.12 R_{\mathrm{E}}$ at $02: 22 \mathrm{UT}$, where $R_{\mathrm{E}}$ denotes the Earth radius. The $y$ component remained steady at $\sim 8.5 R_{\mathrm{E}}$ throughout this interval.

The inferred stability of the shock motion and the slow motion of the spacecraft relative to the shock (from the usual scaling of the foot length with $M_{\mathrm{A}}$ ) are responsible for the longlasting traverse of the foot. There are some indications of minor movement in the overall shock position, but, on the whole, the foot transition in the ion moments is monotonic. In this case, therefore, the slow traverse of the foot implies that the intrinsic time variability can be observed, together with any changes due to convection/propagation. The overall time series is thus a combination of the spatial profile at large scales, and temporal variability together with convection/propagation effects at smaller scales.

The slow decrease in $v_{x}$ and the substantial increase in $v_{y}$ and $T_{\perp}$ over time indicate a strong component of ions reflected at the shock, which changes the center of mass and acts to slow down the incoming solar wind. A $16 \mathrm{~s}$ modulation period is evident in both the ion and magnetic field data, which is equivalent to 0.25 of the upstream proton gyroperiod. This period of modulation is consistent with the time taken for a specularly reflected ion to traverse the foot and return to the shock transition, in agreement with both predictions and previous observations (Schwartz et al. 1983; Sulaiman et al. 2015). In the ion data this modulation is strongest at the upstream edge of the foot, where there is an almost binary pulsing of the reflected component, seen clearly in $T_{\perp}$ and $v_{y}$. Closer to the shock, the periodicity is not as evident, as the contribution from the background level of reflected ions becomes dominant over the solar wind (as measured). This is also evidence that the shock is not undergoing a full reformation where the reflection shuts off and the shock redevelops.

The same periodicity is seen in the magnetic field near the shock ( 01:56-02:03 UTC). The magnetic pulsations are initiated as small perturbations on the background magnetic field $(\Delta B / B \leqslant 1)$, caused by the slowing down of the incident upstream flow by the reflected ions. However, as the particle distribution becomes unstable closer to the shock, bursty pulses are formed by the growth of high-frequency waves with a characteristic frequency of $1-2 \mathrm{~Hz}$. The field amplitude increase within these pulses is almost entirely due to the short period waves, so they do not indicate encounters with the main shock transition. An example from 2013 March 24 is shown in Figure 2. In this event, the close separation of $\mathrm{C} 3$ and $\mathrm{C} 4$ (36 km in the $x$-direction) allows the wave velocity to be estimated. The calculation is based on the time delay between the two spacecraft, determined by a correlation analysis, and an assumption on the wave propagation direction and the orientation of the wave front, as these cannot be fully determined with only two spacecraft. However, it is reasonable to believe that the wave propagation direction is related to the solar wind velocity, with the waves being swept back across the spacecraft with the bulk flow, or that they are roughly aligned with the shock normal in the case of outward propagating waves (or near-phase standing waves). As C3, which is farthest from the shock, always observes the wave signatures ahead of $\mathrm{C} 4$, the first option is the most likely interpretation and the wave velocity can thus be estimated from the $x$-projection of the separation vector and the time delay between the observations.

Due to the short temporal separation between the two spacecraft, the velocity resolution is restricted to $\Delta v \approx 100 \mathrm{~km} \mathrm{~s}^{-1}$, if the time shift is kept to whole samples. However, as the two wave forms are remarkably similar, we can use a sub-sampling by linear interpolation to allow more precise definitions of the time delay. This improves the velocity resolution, and it thus helps provide a better estimate of actual time delay. This analysis results in a propagation velocity of $\sim 200 \mathrm{~km} \mathrm{~s}^{-1}$ in the $-x$-direction, which is close to the background ion flow speed. This provides evidence that the waves are moving toward the shock roughly with the bulk flow. For a characteristic frequency of $2 \mathrm{~Hz}$, the associated flowaligned wavelength is $\sim 100 \mathrm{~km}$, to be compared to the $1400 \mathrm{~km}$ size of the foot. This means that the wavelength of the bursty pulsations are at or below the thermal ion gyroradius and the ion inertial length of the upstream plasma, thus at subproton scales. Examination of data from the Cluster search coil magnetometer STAFF (Cornilleau-Wehrlin et al. 2003) shows that the frequency spectrum of these waves extends far above the proton gyrofrequency to the regime where electron dynamics is important. A minimum variance analysis of the FGM magnetic field data yields a ratio of maximum to intermediate eigenvalue of $\sim 5$, indicating that the perturbations are nearly linearly polarized and perpendicular to the background magnetic field.

Two 2D planar cuts through the ion velocity space distribution function are shown in Figure 3, one within and one outside of a pulse. These velocity cuts are derived from the 3D data in a manner identical to that described by Sundberg et al. (2016): the angle- and energy-dependent sampling points are interpolated onto a Cartesian grid using a nearest neighbor interpolation. This interpolation scheme conserves the bin distribution, and the interpolated data are then rotated from the spacecraft frame into the appropriate reference frame, which allows $2 \mathrm{D}$ cutting planes to be taken at arbitrary velocities and orientations. Qualitatively, the two distributions shown are similar, showing characteristics that are typical for a broad population of ions reflected from and returning to the shock, in agreement with the trajectory expected from gyration in the upstream magnetic field starting from the point of initial reflection. Very near the shock transition, the ratio between the reflected and the upstream solar wind ion flux varies between 0.15 and 0.4 . 


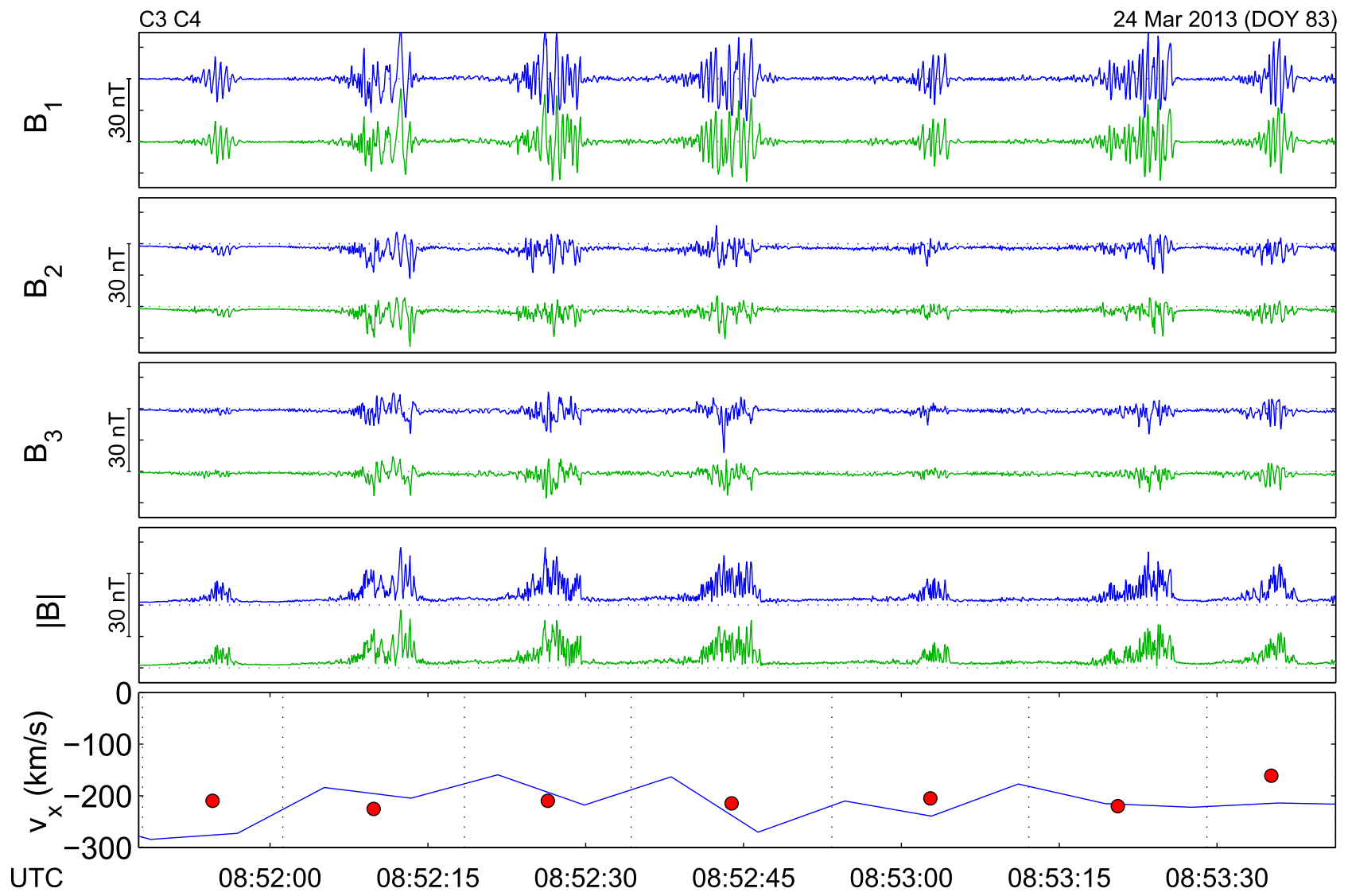

Figure 2. Magnetic field waves observed in the shock foot on 2013 March 24 by C3 (green) and C4 (blue). The two data sets have been offset by $30 \mathrm{nT}$ to facilitate viewing, and the zero baselines are indicated by the dotted lines. The top four panels show the maximum $\left(B_{1}\right)$, intermediate $\left(B_{2}\right)$, minimum $\left(B_{3}\right)$ variance components, and the magnetic field magnitude. The bottom panel shows the $x$-component of the ion velocity, with the phase velocity estimate from $\mathrm{C} 3$ and $\mathrm{C} 4$ marked by the red circles. The dotted lines in the bottom panel indicate the boundaries of the correlation periods used for each of the velocity estimates.

Figure 4 shows the electron particle flux over the same period at eight different sampling points, together with reference distributions of the upstream and downstream spectra. The electron flux densities are typically at magnetosheath-like levels in the higher energy span, and at solarwind-like levels in the lower energy span, although with the low-energy distribution shifted toward the right, consistent with effects of the shock potential (Lefebvre et al. 2007). All spectra vary within the limits of the upstream and downstream references, with no indications that the pulsations lead to any unusual electron energization. The electron heating contributes less than half the total heating at the shock, which agrees with previous statistical studies (Schwartz et al. 1988; Masters et al. 2011).

\section{Discussion and Conclusions}

The results presented here show that the structure of the very high Mach number shock is modulated periodically at a fraction of the upstream ion gyroperiod. The modulation consists of bursty magnetic pulsations with waves at sub-proton scales that are convected toward the shock, growing in amplitude as they approach the shock transition. The waves are linearly polarized and appear to be close to non-propagating in the plasma flow frame. In the reflected ions the modulation is seen most strongly at the upstream edge of the foot, corresponding to ions that have been specularly reflected. However, ion reflection is never fully suppressed, and the shock does not undergo reformation, as usually understood.
There are different models used to discuss the nonstationarity or reformation of high Mach number shocks. The most commonly referred to model is associated with a quasiperiodic switching on and off of ion reflection, where the reflected fraction is greater than required for a stable shock, so that the magnetic field steepens where the reflected ions begin to turn around, leading to a reformation of the abrupt shock transition at a new position (e.g., Lembège \& Dawson 1987; Hada et al. 2003). From Figure 1 it can be seen that the ion perpendicular temperature $T_{\perp}$ is elevated and steady from the shock ramp (at 01:56 UT) out into the foot over many cycles in the periodic magnetic pulsations. The elevated ion perpendicular temperature is due to reflected ions as seen, for example, in Figure 3, and throughout this period the ion velocity space shows a signature of both outward and returning reflected ions. Although there is some modulation in density, velocity and temperature associated with the magnetic pulsations, the reflected ions are present at all times. Thus, ion reflection is never full suppressed by the quasi-periodic behavior of the shock, and the shock does not undergo reformation as understood in the most common model. However, at the upstream edge of the foot (Figure 1, 02:10-02:18 UT) $T_{\perp}$ is strongly modulated, although the velocity is barely changed from its far upstream value. The reflected gyrating ions responsible for the enhanced $T_{\perp}$ here have their turnaround point furthest from the shock ramp (i.e., at the upstream edge of the foot), and have experienced reflection that has given them a perpendicular velocity larger than that of other reflected ions. 


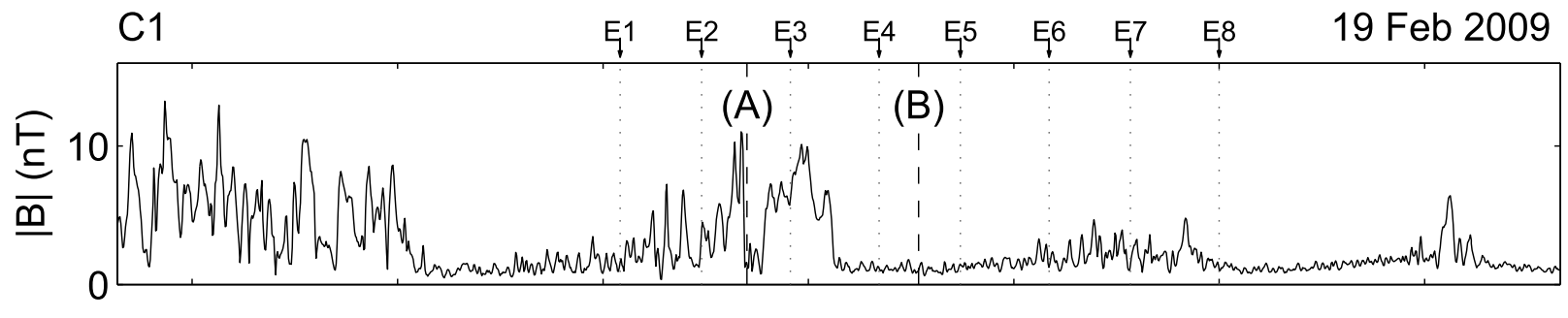

UTC $\quad 01: 56: 10 \quad 01: 56: 20 \quad 01: 56: 30 \quad 01: 56: 40 \quad 01: 56: 50 \quad 01: 57: 00 \quad 01: 57: 10$
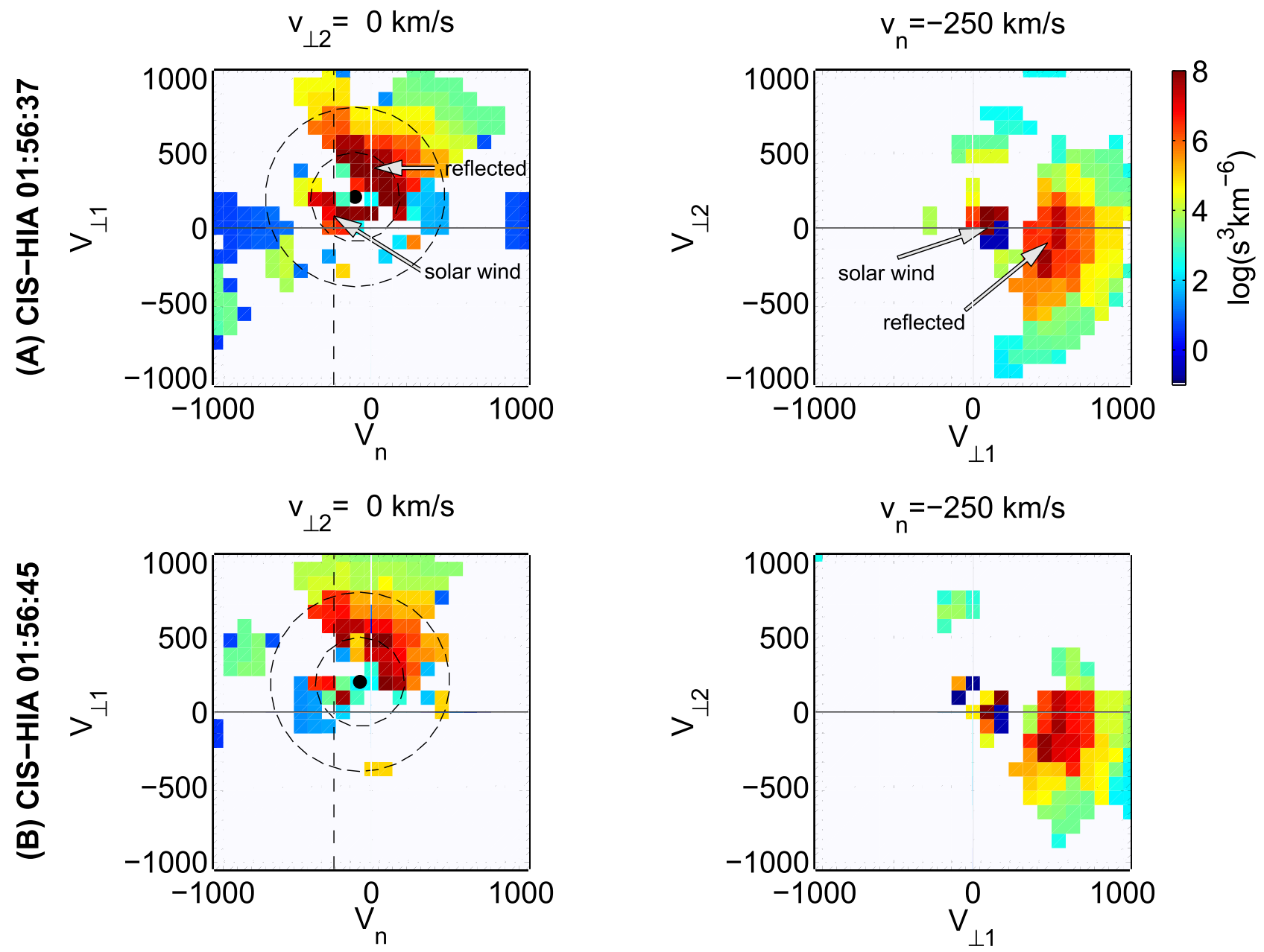

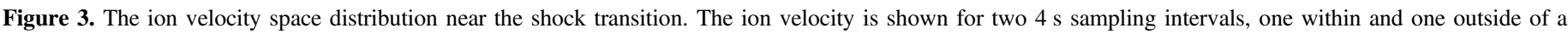
magnetic pulse, marked by (A) and (B) in the top panel. The figure uses a shock-normal coordinate system, where $v_{n}$ is aligned with the shock normal, $v_{\perp 1}$ is perpendicular to the upstream magnetic field, and $v_{\perp 2}$ completes the right-handed system. For each of these two intervals, two different planar cuts are shown: the $v_{n}-$ $v_{\perp 1}$ plane at $v_{\perp 2}=0$ (left column), and the $v_{\perp 1}-v_{\perp 2}$ plane at $v_{n}=-250$ (right column), which cuts near the center of the solar wind beam. As the shock is nearly perpendicular, the magnetic field is roughly parallel to the negative $B_{\perp 1}$-axis, and the $v_{n}-v_{\perp 1}$ cuts are representative of the gyrational component of the velocity. In the left panels, the black spot marks the center of mass, and the concentric circles show sample gyromotion trajectories in the center of mass frame for ion velocities of 300 and $600 \mathrm{~km} \mathrm{~s}^{-1}$. In the magnetic field panel, E1-E8 also marks the time of the electron spectra in Figure 4.

They make only a small contribution to the density, as evident from the lack of velocity change in that region. So, the strong modulation in $T_{\perp}$ is evidence of a modulation in a small fraction of reflected ions that are closest to be being specularly reflected (giving them the largest possible perpendicular velocity). The majority of reflected ions are weakly modulated, and appear to be reflected "non-specularly" (Sckopke et al. 1983) with turnaround positions over a range of distances from the shock ramp, as evidenced by the relatively steady change in velocity toward the shock ramp.
The shock characteristics are also in conflict with other previous theories of shock non-stationarity. The time evolution, which shows a causality in the modulation of the reflected ion density, the slowing down of solar wind, and the triggering of sub-proton scale instabilities, shows that the wave growth is initiated in the foot. This contradicts both whistler-induced (Biskamp \& Welter 1972b; Scholer \& Burgess 2007) and gradient catastrophe models (Krasnoselskikh et al. 2002), which require waves propagating away from the shock transition. The wave properties are also inconsistent with the 

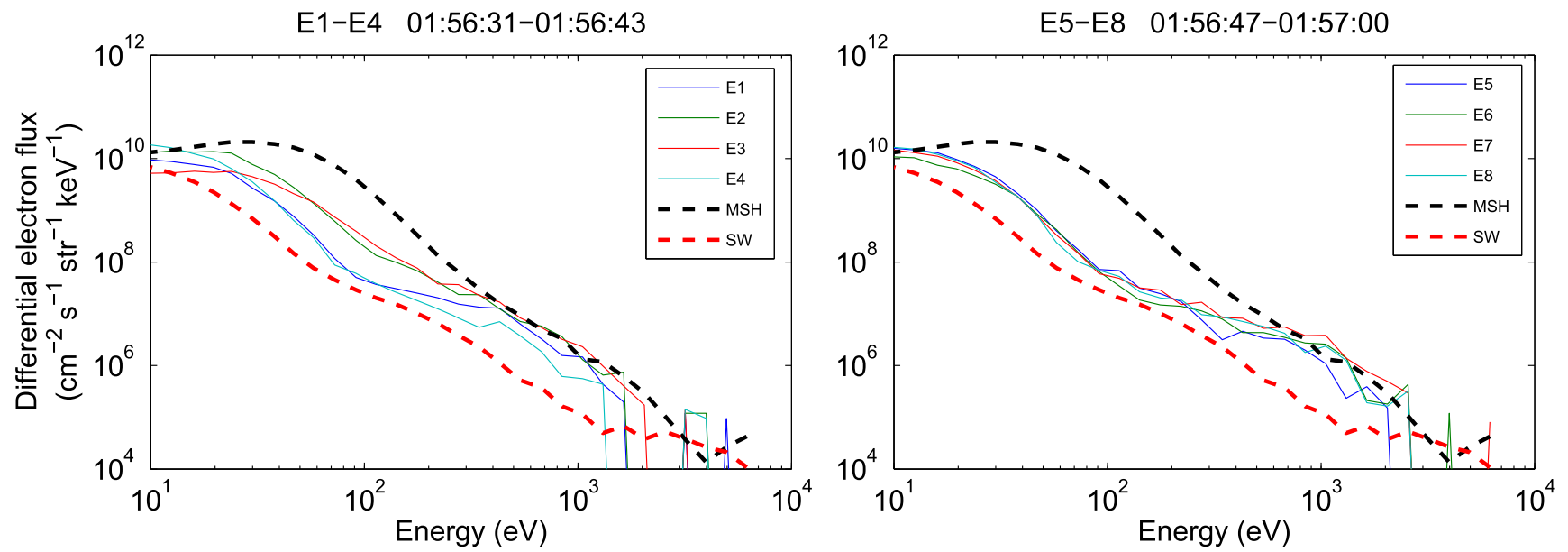

Figure 4. The differential electron particle flux is shown for the eight sampling periods (E1-E8) indicated in Figure 3. The red and black dashed lines indicate the average upstream (solar wind) and downstream (magnetosheath) distributions.

Buneman and the modified two-stream instabilities, with strongly linear waves, and no clear signs of electron bulk heating. In addition, the plasma conditions are Buneman stable, and as the Mach number of the shock exceeds that of the nonlinear critical whistler by an order of magnitude, it is highly unlikely that nonlinear whistlers can propagate upstream from the shock. For these reasons, the modified two-stream instability is not expected to develop, as it is destabilized only when the reflected ion beam velocity is less than the maximum phase velocity of the whistler waves in the electron rest frame.

The wave properties are instead consistent with the ion Weibel instability, a streaming instability that develops between unmagnetized ions and magnetized electrons due to the cross-field current carried by the reflected ions as they gyrate in the foot. This instability has been associated with magnetic field generation in relativistic shocks (Nishikawa et al. 2005; Spitkovsky 2007), but numerical simulations also show that it can produce non-propagating, linearly polarized magnetic field waves in the foot of quasi-perpendicular shocks (Matsukiyo \& Scholer 2006a; Burgess et al. 2016). The effects of the ion Weibel instability in a high Mach number shock were also discussed recently by Matsumoto et al. (2015).

The observations shown here indicate that a coupling between the growth of the ion Weibel instability and the reflection of ions can be a source of large-scale nonstationarities at very high Mach number shocks. Whistler waves are likely to play an important role only within the main shock transition, where the bulk velocity slows.

These results are not in direct contradiction to previous simulations, as the main instabilities influencing the shock dynamics are dependent on the shock parameters, and the current proposed mechanism may primarily be important for high ion $\beta$ shocks. Nevertheless, as the simulations are always subject to restrictions on the physical parameters, these findings show the importance of observational validation. Compromises on the mass ratio between the ions and electrons and the ratio between the plasma frequency and the gyrofrequency will affect the physics captured within the simulation environment (Krasnoselskikh et al. 2013), such as, for example, instability growth rates. A restriction on the spatial dimensions in the simulation can likewise have an important effect. In order to adequately capture the influence of the ion Weibel instability a $2 \mathrm{D}$ simulation with sub-ion-scale resolution is required as a minimum (Burgess et al. 2016). This parameter range is in between the domains typically targeted by hybrid (particle ions with fluid electrons) and full-particle simulations. For these reasons, the restriction imposed by the 1D setup used, for example, in the models by Scholer et al. (2003) and Matsukiyo \& Scholer (2003), may thus exclude the ion Weibel instability in favor of the modified two-stream and Buneman instabilities. Hybrid simulations in 1D at high Mach number $\left(M_{\mathrm{A}} \sim 23\right)$ show some, but not perfect, agreement with non-stationarity observed at the Uranian bow shock (Tiu et al. 2011). However, the maximum field compression seen in the simulations depends directly on the chosen resistivity. This, together with the results shown here, implies that any use of hybrid simulations, particularly one-dimensional simulations, for the very high Mach number regime requires careful validation.

Finally, consistent with earlier observations, the observed shocks have no signs of strong electron acceleration, which is likely explained by the relatively modest electron sonic Mach number of 8 . When considering particle acceleration at astrophysical shocks, it may thus be crucial to select the appropriate Mach number to characterize the shock strength.

This work was supported by the UK Science and Technology Facilities Council (STFC) grants ST/J001546/1 and ST/M001202/1. The research leading to the results presented has received funding from the European Commission's Seventh Framework Programme FP7 under the grant agreement SHOCK (project number 284515). All instrument data are publicly available at the Cluster Science Archive (http://www.cosmos.esa.int/web/csa). We thank the Cluster FGM, CIS, and PEACE instrument teams and the Cluster Science Archive for providing the data used in this study. We also thank NASA/GSFC's Space Physics Data Facility's OMNIWeb service (http://spdf.gsfc.nasa.gov) for providing solar wind data.

\section{References}

Balogh, A., Carr, C. M., Acuna, M., et al. 2001, AnGeo, 19, 1207

Bell, A. R. 2013, APh, 43, 56

Biskamp, D., \& Welter, H. 1972a, NucFu, 12, 663

Biskamp, D., \& Welter, H. 1972b, JGR, 77, 6052

Burgess, D., Hellinger, P., Gingell, I., \& Trávníček, P. M. 2016, JPIPh, 82, 905820401 
Burgess, D., \& Scholer, M. 2015, Collisionless Shocks in Space Plasmas: Structure and Accelerated Particles (Cambridge: Cambridge Univ. Press) Cargill, P., \& Papadopoulos, K. 1988, ApJL, 329, L29

Cornilleau-Wehrlin, N., Chanteur, G., Perraut, S., et al. 2003, AnGeo, 21, 437

Hada, T., Oonishi, M., Lembège, B., \& Savoini, P. 2003, JGR, 108, 1233

Helder, E. A., Vink, J., Bykov, A. M., et al. 2012, SSRv, 173, 369

Johnstone, A. D., Alsop, C., Burge, S., et al. 1997, SSRv, 79, 351

Jokipii, J. R. 2013, SSRv, 176, 115

Krasnoselskikh, V., Balikhin, M., Walker, S. N., et al. 2013, SSRv, 178, 535

Krasnoselskikh, V., Lembege, B., Savoini, P., \& Lobzin, V. 2002, PhP1, 9, 1192

Lefebvre, B., Schwartz, S. J., Fazakerley, A. F., \& Décréau, P. 2007, JGR, 112,9212

Lembège, B., \& Dawson, J. M. 1987, PhFl, 30, 1767

Lobzin, V. V., Krasnoselskikh, V. V., Bosqued, J.-M., et al. 2007, GeoRL, 34 L05107

Masters, A., Schwartz, S., Henley, E., et al. 2011, JGR, 116, A10107

Matsukiyo, S., \& Scholer, M. 2003, JGR, 108, 1459

Matsukiyo, S., \& Scholer, M. 2006a, JGR, 111, A06104
Matsukiyo, S., \& Scholer, M. 2006b, AdSpR, 38, 57

Matsumoto, Y., Amano, T., \& Hoshino, M. 2013, PhRvL, 111, 215003

Matsumoto, Y., Amano, T., Kato, T., \& Hoshino, M. 2015, Sci, 347, 974

Moullard, O., Burgess, D., Horbury, T., \& Lucek, E. 2006, JGR, 111, A09113

Nishikawa, K.-I., Hardee, P., Richardson, G., et al. 2005, ApJ, 622, 927

Reme, H., Bosqued, J. M., Sauvaud, J. A., et al. 1997, SSRv, 79, 303

Russell, C. T. 1985, Planetary Bow Shocks (Washington, DC: American Geophysical Union)

Scholer, M., \& Burgess, D. 2007, PhPl, 14, 072103

Scholer, M., Shinohara, I., \& Matsukiyo, S. 2003, JGR, 108, 1014

Schwartz, S. J., Thomsen, M. F., Bame, S. J., \& Stansberry, J. 1988, JGR, 93, 12923

Schwartz, S. J., Thomsen, M. F., \& Gosling, J. T. 1983, JGR, 88, 2039

Sckopke, N., Paschmann, G., Bame, S. J., Gosling, J. T., \& Russell, C. T. 1983, JGR, 88, 6121

Spitkovsky, A. 2007, ApJL, 673, L39

Sulaiman, A., Masters, A., Dougherty, M., et al. 2015, PhRvL, 115, 125001

Sundberg, T., Haynes, C. T., Burgess, D., \& Mazelle, C. X. 2016, ApJ, 820, 21

Tiu, D., Cairns, I. H., Yuan, X., \& Robinson, P. A. 2011, JGR, 116, A04228 